\title{
Excitation rates of heavy quarks
}

\author{
C. A. Garcia Canal and E. M. Santangelo \\ Laboratorio de Física Teorica, Universidad Nacional de La Plata, 1900 La Plata, Republica Argentina
}

M. B. Gay Ducati

Instituto de Física, Universidade Federal do Rio Grande do Sul, 90000 Porto Alegre, Rio Grande do Sul, Brasil

(Received 9 November 1984)

\begin{abstract}
We obtain the production rates for $c, b$, and $t$ quarks in deep-inelastic neutrino- (antineutrino-) nucleon interactions, in the standard six-quark model with left-handed couplings. The results are obtained with the most recent mixing parameters and we include a comparison between quark parametrizations. The excitations are calculated separately for each flavor, allowing the understanding of the role of threshold effects when considered through different rescaling variables.
\end{abstract}

\section{INTRODUCTION}

The question of production rates of heavy quarks in high-energy neutrino beams is still an object of study and its interest is made evident by the several experiments taking place with such beams in different laboratories. ${ }^{1}$ From the theoretical point of view there exist open problems, since, for example, no good explanation is available for the same-sign-dileptons rates. ${ }^{2}$

In this paper, we present a study of the rates of $c-, b-$, and $t$-quark production in deep-inelastic neutrino-nucleon and antineutrino-nucleon interactions. Our results were obtained using the standard six-quark model with lefthanded couplings and employing the most recent values of the Kobayashi-Maskawa mixing angles, ${ }^{3}$ which are quite stringent concerning $u b$ and $c b$ couplings.

We present curves of excitation of heavy quarks distinguishing all the possible channels of production, calculated with two different QCD-dependent parametrizations ${ }^{4,5}$ of quark distributions and a simple quark-parton distribution. ${ }^{6}$ The evolution until the free hadron states originated at the quark level could not be completely explained without the understanding of the fragmentation mechanism. In order to test fragmentation models, it is clear that one needs a complete parametrization able to cover properly the quark-excitation mechanism up to high energies.

To have a feeling about the regions of dominance of the production of the different flavors and to estimate future rates, results are presented for energies as high as 1200 $\mathrm{GeV}$, even though the most recent experimental data attain $250 \mathrm{GeV}$.

The aim of this work is to estimate, constrained to the recent values for the parameters involved, at which energies new effects should be expected due to the excitation of heavy quarks. The analysis concerning the top quark is included because it seems that this flavor is showing up in the very recent UA1 experiment. ${ }^{7}$

In Sec. II we present the cross-section formulas in order to fix our notation. In Sec. III we discuss the quarkdistribution parametrizations used in our predictions. In Sec. IV we present the very recent mixing parameters used. In Sec. $V$ we discuss the fundamental role of rescaling, the variable which takes into account threshold effects, in the calculation. In Sec. VI we present the results, together with some comments.

\section{CROSS SECTIONS}

The differential cross section for neutrino-nucleon (isoscalar target) interaction is given in terms of the CallanGross structure functions as

$$
\begin{aligned}
\frac{d^{2} \sigma^{v}}{d x d y}=K & {\left[\left(\frac{x y}{\xi}+(1-y)\right] F_{2}(\xi)\right.} \\
& \left.+\left(\frac{x}{\xi}\left(y^{2}-y\right)+(1-y)\right] \bar{F}_{2}(\xi)\right],
\end{aligned}
$$

and for antineutrino-nucleon interaction as

$$
\begin{aligned}
\frac{d^{2} \sigma^{\bar{v}}}{d x d y}=K[ & {\left[\frac{x}{\xi}\left(y^{2}-y\right)+(1-y)\right] F_{2}(\xi) } \\
& \left.+\left(\frac{x y}{\xi}+(1-y)\right] \bar{F}_{2}(\xi)\right],
\end{aligned}
$$

where $K=G^{2} M E / \pi, G$ being the Fermi constant, $M$ the nucleon mass, and $E$ the neutrino (antineutrino) incident energy; $x$ and $y$ are the scaling variables, and $\xi$ the rescaling variable, introduced in order to take properly into account threshold effects, as will be discussed later. $F_{2}$ and $\bar{F}_{2}$ are the structure functions, related to quarks and antiquarks, respectively.

In terms of quark distributions, expressions (2.1) and (2.2) give rise to

$$
\begin{aligned}
\frac{d^{2} \sigma^{\nu N \rightarrow l-X}}{d x d y}=K \xi[ & {\left[(1-y)+\frac{x y}{\xi}\right]\left\{[u(\xi)+d(\xi)] V_{d}^{2}++2 s(\xi) V_{s}^{2}+2 b(\xi) V_{b}^{2}\right\} } \\
& \left.+\left[(1-y)+\frac{x}{\xi}\left(y^{2}-y\right)\right]\left\{[\bar{u}(\xi)+\bar{d}(\xi)] V_{u}^{2}+2 \bar{c}(\xi) V_{c}^{2}+2 \bar{t}(\xi) V_{t}^{2}\right\}\right]
\end{aligned}
$$


and

$$
\begin{aligned}
\frac{d^{2} \sigma^{\bar{v} N \rightarrow l^{+} X}}{d x d y}=K \xi & {\left[(1-y)+\frac{x}{\xi}\left(y^{2}-y\right)\right]\left\{[u(\xi)+d(\xi)] V_{u}{ }^{2}+2 c(\xi) V_{c}^{2}+2 t(\xi) V_{t}^{2}\right\} } \\
& \left.+\left[(1-y)+\frac{x y}{\xi}\right]\left\{[\bar{u}(\xi)+\bar{d}(\xi)] V_{d}{ }^{2}+2 \bar{s}(\xi) V_{s}{ }^{2}+2 \bar{b}(\xi) V_{b}{ }^{2}\right\}\right],
\end{aligned}
$$

where $u(\xi), d(\xi)$, etc., are the quark distribution functions, depending on the rescaling variable $\xi$, and $V_{i}^{2}$ is the sum of a row or column of the Kobayashi-Maskawa matrix, so

$$
\begin{aligned}
& V_{u}^{2}=V_{u d}^{2}+V_{u s}^{2}+V_{u b}^{2}, \\
& V_{d}^{2}=V_{u d}^{2}+V_{c d}^{2}+V_{t d}^{2}, \\
& V_{i}^{2}=1 .
\end{aligned}
$$

The partial productions are immediately obtained from the general cross-section expressions, Eqs. (2.3) and (2.4), since the couplings are obtained from

$$
\left(\begin{array}{lll}
\bar{u} & \bar{c} & \bar{t}
\end{array}\right)\left(\begin{array}{lll}
V_{u d} & V_{u s} & V_{u b} \\
V_{c d} & V_{c s} & V_{c b} \\
V_{t d} & V_{t s} & V_{t b}
\end{array}\right)\left(\begin{array}{l}
d \\
s \\
b
\end{array}\right),
$$

and so we have, for instance,

$$
\begin{aligned}
& \left.\frac{d \sigma^{v N \rightarrow \mu^{-} c X}}{d x d y}=K \xi\left[(1-y)+\frac{x y}{\xi}\right]\left\{[u(\xi)+d(\xi)] V_{c d}{ }^{2}+2 s(\xi) V_{c s}{ }^{2}+2 b(\xi) V_{c b}{ }^{2}\right\}\right], \\
& \left.\frac{d \sigma^{\bar{v} N \rightarrow \mu^{+} \bar{t} X}}{d x d y}=K \xi\left[(1-y)+\frac{x y}{\xi}\right]\left\{[\bar{u}(\xi)+\bar{d}(\xi)] V_{t d}{ }^{2}+2 \bar{s}(\xi) V_{t s}{ }^{2}+2 \bar{b}(\xi) V_{t b}{ }^{2}\right\}\right], \\
& \left.\frac{d \sigma^{\bar{v} N \rightarrow \mu^{+} b X}}{d x d y}=K \xi\left[(1-y)+\frac{x}{\xi}\left(y^{2}-y\right)\right]\left\{[u(\xi)+d(\xi)] V_{u b}{ }^{2}+2 c(\xi) V_{c b}{ }^{2}+2 t(\xi) V_{t d}{ }^{2}\right\}\right] .
\end{aligned}
$$

The quark distribution functions depend on the rescaling variable, and when considering QCD-dependent parametrizations, on the QCD parameters $\Lambda$ and $Q^{2}$.

\section{QUARK DISTRIBUTION FUNCTIONS}

Our results were obtained from three different parametrizations for quark distribution functions. The first QCD-based parametrization is the standard BurasGaemers one ${ }^{4}$ and the second one considered is that recently proposed in Ref. (5), whose parameters include a second-order dependence on the QCD evolution parameter

$$
\bar{s}=\ln \left[\ln \left(Q^{2} / \Lambda^{2}\right) / \ln \left(Q_{0}{ }^{2} / \Lambda^{2}\right)\right],
$$

having the general form for valence quarks,

$x\left[u_{V}\left(x, Q^{2}\right)+d_{V}\left(x, Q^{2}\right)\right]=C_{1} x^{\eta_{1}}(1-x)^{\eta_{2}}\left(1+\gamma_{1} x\right)$, (3.1a) and for $\mathrm{SU}(3)$-symmetric sea,

$x S\left(x, Q^{2}\right)=A_{S} x^{a_{S}}(1-x)^{b}\left(1+\alpha_{S} x+\beta_{S} x^{2}+\gamma_{S} x^{3}\right)$.

These QCD parametrizations are obtained by fixing parameters both from the momentum sum rules and fitting from available data. We expect that the results obtained using Ref. 5 could be more predictable, since that parametrization was constructed using more recent experimental data, which covers energy values up to $200 \mathrm{GeV}$.

As a matter of comparison we also present results from a naive quark-parton parametrization; ${ }^{6}$ since we are interested in the high-energy domains, this permits us to separate scaling violation from QCD effects and threshold

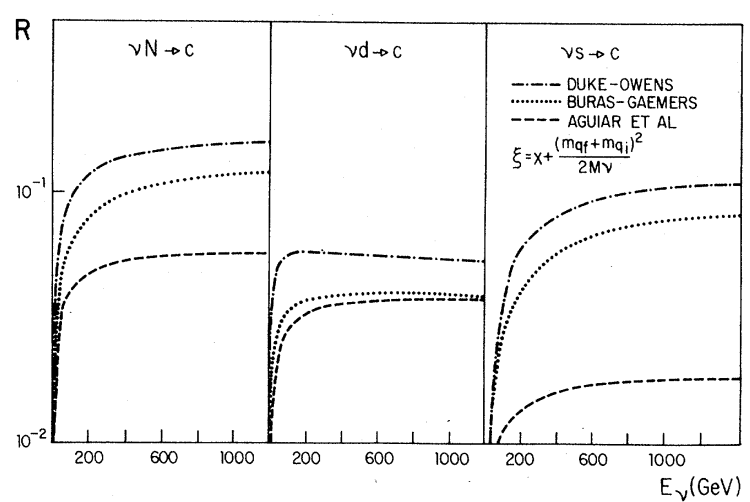

FIG. 1. Total and partial production rates of $c$ quarks from neutrino-nucleon interactions for $E_{v}$ up to $1200 \mathrm{GeV}$, obtained with three different quark parametrizations. The QCD parameters are $\Lambda^{2}=0.25 \mathrm{GeV}^{2}$ and $Q_{0}{ }^{2}=1.8 \mathrm{GeV}^{2}$ for Ref. 4 and $\Lambda^{2}=0.16 \mathrm{GeV}^{2}$ and $Q_{0}{ }^{2}=4 \mathrm{GeV}^{2}$ for Ref. 5, and $m_{c}=1.9 \mathrm{GeV}$. 
effects coming with the rescaling. This can be seen in Fig. 1, for charm excitation.

\section{MIXING PARAMETERS}

Due to the large amount of data coming from different experiments, providing more and more constraints, the mixing parameters are a subject of continuous analysis and discussion, reaching more precise and, in some cases, more stringent values. Very low values seem to be confirmed for the off-diagonal terms of the mixing matrix related to the $b$-quark couplings $V_{u b}$ and $V_{c b}$. The experimentally determined large $B$ lifetime, ${ }^{8,9}$ with values around 1 psec, has a direct relation in those parameters since the $b$-quark lifetime is given by ${ }^{10}$

$$
\frac{1}{\tau_{b}}=\frac{G_{F} m_{b}^{5}}{192 \pi^{2}}\left(2.95\left|V_{c b}\right|^{2}+6.33\left|V_{u b}\right|^{2}\right),
$$

and so values lower than the previous ones are obtained for $V_{u b}$ and $V_{c b}$. Such low values for the mixing parameters strongly suppressed the theoretical predictions for same-sign-dilepton production rates via $b$ production. ${ }^{11}$

In our calculation we considered the most recent set of mixing parameters given by ${ }^{3}$

$$
\left(\begin{array}{lll}
0.945 & 0.231 & 0.005 \\
0.1 & 0.972 & 0.044 \\
0.024 & 0.069 & 0.99
\end{array}\right)
$$

in order to have an upper-bound estimation for the production rates for each flavor.

\section{RESCALING}

We face at this point the question of rescaling, which has different formulations ${ }^{12,13}$ as the excitation process occurs from a light-to-light, light-to-heavy, or heavy-toheavy quark. From those formulations we should conclude that the most general one is the slow rescaling of the form

$$
\xi=x+\frac{\left(m_{q_{i}}+m_{q_{f}}\right)^{2}}{2 M v},
$$

where $m_{q_{f}}$ denotes the final-quark mass and $m_{q_{i}}$ the initial-quark mass, since this expression takes into account the production of heavy-to-heavy quarks, when it becomes kinematically allowed. It also reduces to the usual ${ }^{12}$ rescaling variable when $m_{q_{i}}$ is negligible

$$
\xi_{\text {usual }}=x+\frac{m_{q_{f}}{ }^{2}}{2 M v} \text {. }
$$

\section{A squared threshold invariant mass}

$$
W_{\mathrm{th}}^{2}+\left(m_{q_{f}}+m_{q_{i}}\right)^{2}+M^{2}
$$

corresponds to the rescaling (5.1).

The introduction of a rescaling variable, of any type, to take into account the effect of mass thresholds on the structure functions implies a detailed analysis of the $x$ and $y$ variation ranges.

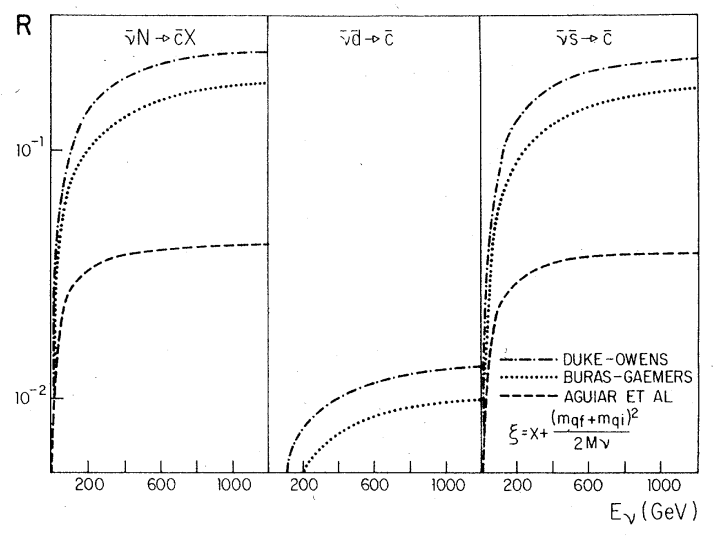

FIG. 2. Total and partial production rates of $\bar{c}$ quarks from antineutrino-nucleon interactions for $E_{\bar{v}}$ up to $1200 \mathrm{GeV}$. The parameters are the same as for Fig. 1.

In any process of quark excitation the invariant mass $W$ recoiling against the scattered lepton must satisfy $W>W_{\text {th }}$, where

$$
W^{2}=M^{2}+2 M E y(1-x) \text {. }
$$

This can be written, in terms of $x$ and $y$, considering the above limit, as

$$
y(1-x) \geq \frac{W_{\mathrm{th}}{ }^{2}-M^{2}}{2 M E},
$$

and so

$$
\begin{aligned}
& E \geq \frac{W_{\mathrm{th}}{ }^{2}-M^{2}}{2 M}, \\
& y \geq \frac{W_{\mathrm{th}}^{2}-M^{2}}{2 M E}, \\
& x \leq 1-\frac{W_{\mathrm{th}}^{2}-M^{2}}{2 M E y},
\end{aligned}
$$

where we can easily see the limitations imposed by $W_{\text {th }}$ on the upper $x$ bound and lower $y$ bound.

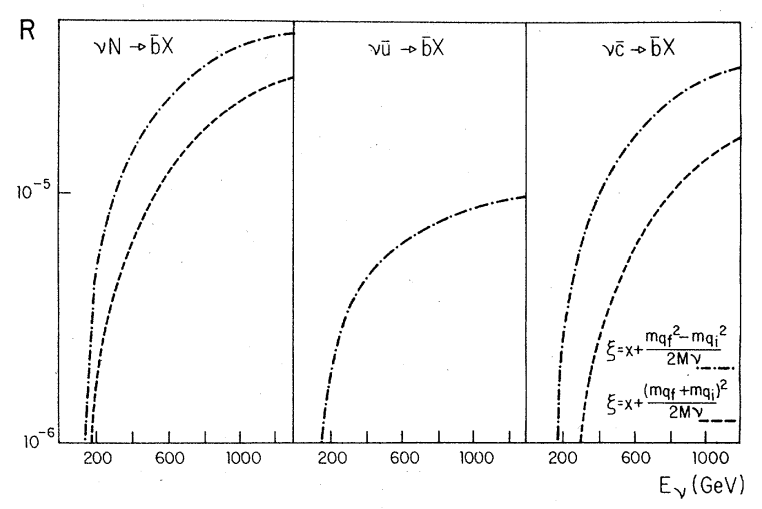

FIG. 3. Total and partial $\bar{b}$ production rates from neutrinos obtained with the parametrization (Ref. 5) showing the effect of different rescalings with $m_{b}=4.9 \mathrm{GeV}$. 


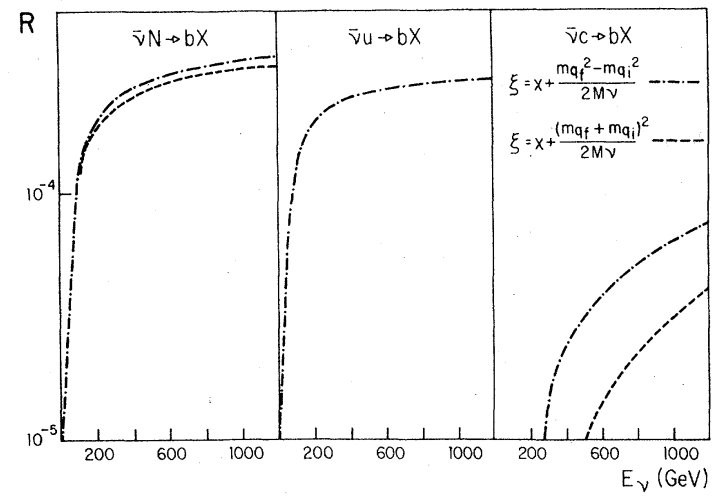

FIG. 4. Total and partial bottom-quark production rates from antineutrinos, calculated with Ref. 5 showing the effect of different rescalings.

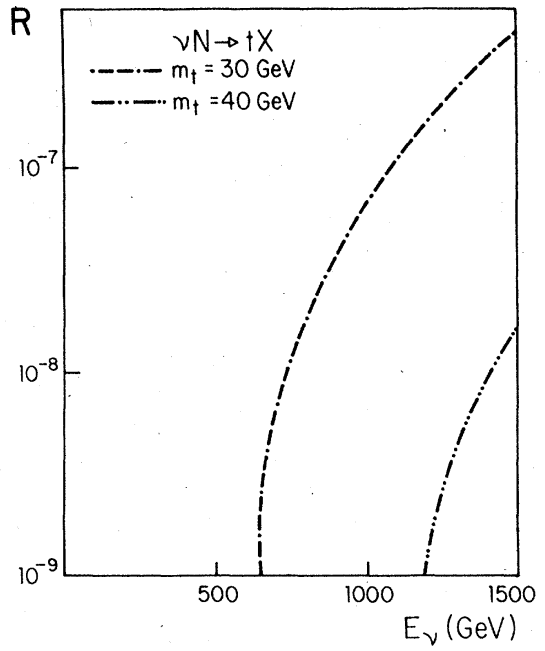

FIG. 5. Total top-quark production rates from neutrinos with $E_{v}$ up to $1500 \mathrm{GeV}$, obtained using parametrization (Ref. 5) for $m_{t}=30$ and $40 \mathrm{GeV}$.

The threshold considerations have a direct consequence in the integral domains ${ }^{14}$ we must calculate in order to obtain the cross sections. It is now clear that the rescaling variable is a function of the masses of all the quarks involved.

The general form of the integration is

$$
R\left(q_{f}\right)=\frac{K}{\sigma_{1 \mu}} \sum_{q_{i}} \int_{\left(m_{q_{f}}+m_{q_{i}}\right)^{2} / 2 M E}^{1} d y \int_{0}^{1-\left(m_{q_{f}}+m_{q_{i}}\right)^{2} / 2 M E y} d x \mathscr{F}\left(x, \xi\left(q_{i}, q_{f}\right), Q^{2}\right) V_{q_{i} q_{f}}{ }^{2}
$$

where we normalize the result in terms of the single muon cross section. The function $\mathscr{F}$ includes the quark distribution functions and multiplicative factors [see Eqs. (2.3) and (2.4)].

\section{RESULTS}

The main purpose of this work is to provide the production rates of heavy quarks in neutrino- and antineutrino-nucleon high-energy interactions, with QCD parametrizations including charm sea distribution but no other heavy sea $(b, t)$ contribution. The reason is that since the charmed sea contribution is probably ${ }^{12}$ as much as $10 \%$ of the whole light sea, the distributions of bottom and top quarks should be strongly suppressed due to their higher masses.

We include in Fig. 1 the partial production rates for $c$ quark, for neutrinos, coming from $d$ and $s$ quarks, and the total rate, comparing the results for the three used parametrizations. In Fig. 2 we present the curves obtained for $\bar{c}$ production by antineutrinos.

Figure 3 shows the bottom rates for neutrinos, and Fig. 4 for antineutrinos, considering the parametrization of Ref. 5 and distinguishing the effects due to different rescaling variables.
In Fig. 5 we include the results obtained for top-quark production rates, considering the top-quark mass at 30 and $40 \mathrm{GeV}$. Notice that for masses greater than $40 \mathrm{GeV}$ the possibility of top-quark-excitation determination in neutrino- (antineutrino-) nucleon interactions could be excluded due to the very low rates at present energies.

All the curves show a smooth behavior with the energy of the incoming neutrino (antineutrino). The importance of threshold effects is clear and for that reason different rescaling variables could give rise to different predictions for bottom-quark excitation rates. It is interesting to note that the effect of the rescaling is much less important in antineutrino bottom-quark production (see Fig. 4) than in the neutrino process (see Fig. 3), because in the former the dominant contribution is from $u$ distribution which is from valence. In the latter case both contributions are from the sea. Predictions for top-quark mass of 30 and $40 \mathrm{GeV}$ are provided (Fig. 5) because these seem to be in the range of values experimentally indicated. ${ }^{7}$

The curve corresponding to charm production from $d$ quarks coming from the parametrization of Ref. 5 shows a slow decreasing for high energies, probably due to parameter-fixing precision, and we think the effect should not be taken seriously. It just shows the need of a parametrization able to cover a larger range of energies, and having simultaneously a good fit at already experimentally covered energy ranges. 


\section{ACKNOWLEDGMENTS}

We would like to acknowledge H. Fanchiotti, H. Vucetich, and C. Camarata for illuminating discussions. One of the authors (M.B.G.D.) is very grateful for the kind hospitality of the Theoretical Physics Department of La Plata
University. This work was partially supported by Conselho Nacional de Desenvolvimento Científico Tecnológico (CNPq), Brasil, and by Consejo Nacional de Investigaciones Cientificas y Téchnicas (CONICET), Argentina.
${ }^{1}$ C. Baltay et al., Phys. Rev. Lett. 52, 1948 (1984); M. Jonker et al., Phys. Lett. 99B, 265 (1981); J. E. Kim et al., Rev. Mod. Phys. 53, 211 (1981).

${ }^{2} \mathrm{~V}$. Barger, in Proceedings of the 21st International Conference on High Energy Physics, Paris, 1982, edited by P. Petiau and M. Porneuf [J. Phys. (Paris) Colloq. 43, C3 (1984)].

${ }^{3}$ P. Langacker, work presented at XXIInd International Conference on High Energy Physics, Leipzig, 1984 (unpublished).

${ }^{4}$ A. J. Buras and K. J. F. Gaemers, Nucl. Phys. B132, 249 (1978).

${ }^{5}$ D. W. Duke and J. F. Owens, Phys. Rev. D 30, 49 (1984).

${ }^{6}$ C. E. M. Aguiar, C. A. Garcia Canal, and J. A. Martins
Simões, Lett. Nuovo Cimento 36, 69 (1983).

${ }^{7}$ R. Böck, work presented at XXIInd International Conference on High Energy Physics, Leipzig, 1984 (unpublished).

${ }^{8}$ E. Fernandez et al., Phys. Rev. Lett. 51, 1022 (1983).

${ }^{9}$ N. S. Lockyer et al., Phys. Rev. Lett. 51, 1316 (1983).

${ }^{10}$ Ling-Lie Chau and Way-Yee Keung, Phys. Rev. D 29, 592 (1984).

${ }^{11}$ J. Smith and C. H. Albright, Phys. Lett. 85B, 119 (1979).

${ }^{12}$ Choy-Heng Lai, Phys. Rev. D 18, 1422 (1978).

${ }^{13}$ R. J. N. Phillips, Nucl. Phys. B212, 109 (1983).

${ }^{14}$ D. M. Scott and K. Tanaka, Phys. Rev. D 21, 1771 (1980). 